\title{
ZnT10 is expressed in adult human tissues
}

\author{
H. J. Bosomworth ${ }^{1}$, D. Ford ${ }^{2}$ and R. A. Valentine ${ }^{1}$ \\ ${ }^{1}$ School of Dental Sciences, Human Nutrition Research Centre, Newcastle University, Framlington Place, \\ Newcastle Upon Tyne, Tyne and Wear NE2 4BW, UK and ${ }^{2}$ Institute for Cell and Molecular Biosciences, \\ University of Newcastle upon Tyne, The Medical School, Newcastle upon Tyne, NE2 4HH, UK
}

$\mathrm{Zn}$ is an important nutrient for the human subjects' body and essential for many proteins and enzymes. Zn homoeostasis is strictly controlled by transport proteins. ZIP are thought to increase cytosolic Zn concentrations and ZnT are responsible for reducing cytosolic $\mathrm{Zn}$ by acting as effluxers.

ZnT10 has been predicted to be expressed only in fetal tissue ${ }^{(1)}$; however, we detected a restricted pattern of ZnT10 expression at the mRNA level in a range of adult human tissues - cervix, liver, brain, ovary, testes, small intestine and colon - by using real time PCR to screen a human subjects RNA tissue panel (Ambion).

To examine the intracellular distribution of ZnT10, SY5Y cells (human subjects neuroblastoma) were transiently transfected with a plasmid construct from which ZnT10 was expressed with an N-terminal FLAG epitope tag. Forty eight hours post transfection cells were fixed using $4 \%$ paraformaldehyde (PFA) and stained with the anti-FLAG antibody. Dual labelling with markers of specific subcellular compartments was also carried out. ZnT10 showed staining consistent with plasma membrane and Golgi apparatus localisation.

To investigate the effect of extracellular $\mathrm{Zn}$ on $\mathrm{ZnT} 10$ expression at the mRNA level $\mathrm{Zn}$ supplementation studies were carried out using the SY5Y cell line model. Cells were treated with either a basal media, or media supplemented with 50,100 or $150 \mu \mathrm{M} \mathrm{ZnCl}{ }_{2}$ for $24 \mathrm{~h}$. Total RNA was extracted and qRT-PCR was carried out using glyceraldehydes 3-phosphate dehydrogenase (GAPDH) as a house-keeper gene. Cells treated with increasing levels of extracellular Zn showed a significant decrease in ZnT10 expression.

\begin{tabular}{lcccccccc}
\hline & \multicolumn{9}{c}{ Zn treatment } \\
\cline { 2 - 9 } $\begin{array}{l}\text { mRNA levels } \\
\text { expressed as a } \\
\text { ratio of GAPDH }\end{array}$ & Untreated & $50 \mu \mathrm{M}$ & $100 \mu \mathrm{M}$ & $150 \mu \mathrm{M}$ & SE & Mean & SE \\
\cline { 2 - 9 } & Mean & SE & Mean & SE & Mean & $4.15 \dagger$ & 0.89 & $4.71 \dagger$ \\
ZnT10 & 8.20 & 0.62 & $5.79^{*}$ & 0.80 & 0.73 \\
MT1 & 0.06 & 0.01 & 0.12 & 0.02 & $0.27^{*}$ & 0.07 & $0.46 \dagger$ & 0.11 \\
\hline
\end{tabular}

Mean values are stated, $n$ 6-9 for each treatment. $* P<0.05, \uparrow P<0.001$, by one way ANOVA followed by LSD multiple comparisons test compared with untreated cells.

This mode of regulation is in contrast to $\mathrm{ZnT1}$, whose transcriptional regulation has been shown to be up regulated in response to $\mathrm{Zn}$ by the transcription factor MTF-1. ZnT5 also shows a down-regulation in response to $\mathrm{Zn}^{(2)}$. Furthermore, a potentially novel zinc responsive element (ZRE) found in the 5-UTR of ZnT5 has also been identified in ZnT10 with initial investigations indicating a role in the downregulation of ZnT10.

The discovery that ZnT10 is expressed in adult tissue is in contrast to published data ${ }^{(1)}$ and its expression in brain tissue together with its down regulation in response to $\mathrm{Zn}$ at the mRNA level in neuroblastoma cells is particularly interesting due to the hypothesised link between $\mathrm{Zn}$ and neurodegenerative diseases.

This work was supported in part by an MRC studentship to HJB.

1. Seve M, Chimienti F, Devergnas S et al. (2004) BMC Genomics 5, 32.

2. Jackson KA, Valentine RA, Coneyworth LJ et al. (2008) Biochem Soc Trans 36 (Pt 6), 1262-1266. 